\title{
Plasmon-excitonic polaritons in metal-semiconductor nanostructures with quantum wells
}

\author{
(C) V.A. Kosobukin
}

loffe Institute,

194021 St. Petersburg, Russia

E-mail: Vladimir.Kosobukin@mail.ioffe.ru

A theory of plasmon-excitonic coupling and its spectroscopy is developed for metal-semiconductor nanostructures. Considered as a model is a periodic superlattice with cells consisting of a quantum well and a layer of metal nanoparticles. The problem is solved self-consistently using the electrodynamic Green's functions taking account of resonant polarization. Coulomb plasmon-exciton interaction is associated with the dipole surface plasmons of particles and their image charges due to excitonic polarization of neighboring quantum well. Optical reflection spectra are numerically investigated for superlattices with GaAs/AlGaAs quantum wells and silver nanoparticles. Superradiant regime caused by one-dimensional Bragg diffraction is studied for plasmonic, excitonic and plasmon-excitonic polaritons depending on the number of supercells. The plasmon-excitonic Rabi splitting is shown to occur in reflectivity spectra of resonant Bragg structures. 